\title{
INCIDENCES OF DEVELOPING EATING DISOREDERS DUE TO BINGE EATING
}

\author{
Sadaf Ahmed, Shamoon Noushad, Syed Zain Azher \& Shaikh Mohammad Tahir \\ Health Science Research Division-AEIRC \\ Corresponding Author Email: zain@aeirc-edu.com
}

\begin{abstract}
Binge eating or chaotic eating refers to unhealthy eating patterns that are found in low incidences comparatively to verify and diagnosed eating disorders. Altered eating behaviors may lead to severe eating disorders like Binge eating disorder and bulimia nervosa that could be life threaten if left undiagnosed The purpose of this study is to investigate unordered or binge eating attitudes among individuals with respect to their eating patterns. An observational study has been done in which a randomized data of 120 individuals have been selected within a group of 24 males and 96 females with the help of a questionnaire based survey on food habits, preference of type of food intake, tea consumption, munching habits, workplace or home eating and eating during working hours. R Results were analyzed on the basis of percentage values and SPSS 17.0 different associations between variables. out of 120 people 58.3\% of the individuals including both genders did not have a feeling of fullness, $50.83 \%$ of the total individuals eat during working hours and possess munching habit, $83 \%$ use to eat biscuits to munch .Unordered eating patterns also affect the frequency of bowel movements, $58.30 \%$ people have bowel movements once a time. Irregular eating and munching habits does not initiate a feeling of fullness in the beginning of a meal, due to binge eating people do get satisfied by the smell of food and did not take a proper meal to fulfill nourishment requirements. However, binge eating lead to increase appetite as the munching material including snacks digest quickly which cause more food intake and people did not get fully satisfied by the smell of food only and excessive tea intake is one of the contributing factors. Binge eating affects normal appetite, eating attitudes and Bowel movements which may further lead to severe eating disorders like Bulimia nervosa which should be clarified early instead left undiagnosed.
\end{abstract}

\section{KEYWORDS}

Eating disorder, binge eating, bulimia nervosa, craving, appetite.

\section{INTRODUCTION}

Eating large amounts of food in full stomach with no feeling of physiological hunger can lead an individual to eating disorder (Abraham, 2008). To hide or stockpile food for secret eating with no planned mealtimes, restrictive dieting or purging is an abnormal set of behavior that can accelerate by lack of exercise, inadequate sleep and average weight or overweight are the contributing factors which leads towards eating disorders (Mathes, 2009). Eating disorders are considered to be a serious condition with partial success of treatment and high morbidity and co - morbidity with other Health conditions (Zali, 2008). The eating disorder has been described as episodes of uncontrolled eating i.e. Binge Eating. Patients with this condition experience loss of control over their eating which leads to difficult eating behaviors during which large amount of food rapidly consumed (Peter, 1983). Research on binge eating disorder still in the early stage and relationship with other eating disorder mainly bulimia nervosa continue to be debated (Christopher, 1998). Severity of binge eating has been associated with many psychiatric illness binge eaters tend to experience depression and emotional distress (Byron, 1999) with report that negative mood states tend to precede binge episodes and is throughout a binge episode (Kuehnel, 1994) while research has examined the relationship between depression and binge eating, other contributing factors have not been investigated (Byron, 1999).This eating disorder can cause serious physical problems that could be more severe and can even be life-threatening with higher ratio in females than males (Anju, 2012 ; Heather, 2001). Though eating disorders are higher in smokers as compared to nonsmokers (Doris, 2006) While Food craving is another factor which is liable for binge eating in bulimia nervosa, initially in which calorific limitation and homeostatic imbalances cause cravings and drive individuals to binging. Though several studies have downplayed the role of hunger in the experience of food cravings and the impact of dietary self-control (Anthonio, 2003). This abnormal Change in appetite affects Bowel movements. Mostly people have Bowel movement once in a day which is not exactly a standard healthy pattern (Davies, 1998). The low intake of fiber and high intake of dietary fat are interlinked with an increased risk of serious diseases, including digestive system cancers and cardiovascular diseases (Shirley, 1997). Restrictive dieting is an additional factor for binge eating because most of the people unable to adhere to their diets and they report episodes of binge eating (Lowe, 1993). However this binging can be linked or confuse people with eating disorders such as bulimia nervosa in which people often consume large amount of drink containing caffeine similar to tea, as it is believed that caffeine increases metabolic rate and suppresses appetite (Anthony, 2005).

\section{METHODOLOGY}

An observational study has been done in which a randomized data of 120 individuals have been selected from Karachi. Karachi is the capital of province Sindh and city of Pakistan. Age group of this study is 24 males and 96 females with the help of self-generating questionnaire based survey on food habits, preference of the type of food intake, tea consumption, munching habits, workplace or home eating and eating during working. However data was analyzed by statistical software SPSS 17.0. We investigate unordered or binge eating attitudes among individuals with respect to their eating patterns. We also discuss the association between bowel movements and the frequency of food intake at different 
intervals of work hours. Moreover, to figure out particular food

\section{RESULTS}

Results were analyzed on the basis of percentage values and with SPSS 17.0 by using $p$. value through chi-square tests different associations among variables and $58.3 \%\left(\chi^{2}=5.053, \mathrm{p}=0.31\right)$ of the individuals including both genders did not have a feeling of fullness, while $38.33 \%$ feels full early in the beginning of food intake. On the other hand $22.60 \%\left(\chi^{2}=32.143, \mathrm{p}=0.001\right)$ individuals were not influenced by the smell of food and while $71.60 \%\left(\chi^{2}=32.143, p=0.001\right)$ influence of smell of food. 50.83 stuff that mostly individuals consume during munching. $\%\left(\chi^{2}=0.426, p=0.031\right)$ of the total individuals eat during working hours and possess munching habit, out of 120 people $83 \%$ use to eat biscuits while $25.83 \%$ consume chips as their munching material. Due to munching one did not get full satisfaction plus excessive tea consumption twice in a day by $36.66 \%\left(\chi^{2}=32.143\right.$, $\mathrm{p}=0.001$ ) lead to eat more. Mostly about $85.83 \%$ preferred to take meals at home and $52.50 \%$ dine out once or twice in a month. Unordered eating patterns also affect the frequency of bowel movements, $58.30 \%\left(\chi^{2}=70.337, \mathrm{p}=0.001\right)$ people have bowel movements once a time whereas $31.60 \%$ had twice daily while $4.16 \%$ had more than three times a day.

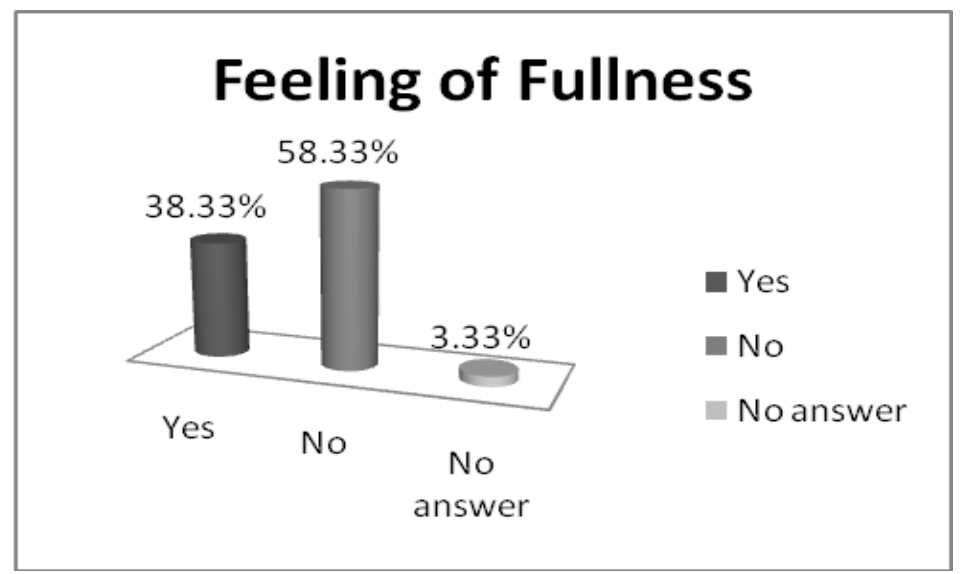

Figure 1: This figure shows reply of 120 peoples concerning the feeling of fullness in the beginning of food intake, of which $\mathbf{5 8 . 3 \%}$ of the individuals including both genders did not have a feeling of fullness, while $38.33 \%$ feels full early in the beginning of food intake $\& 3.33 \%$ give no answer.

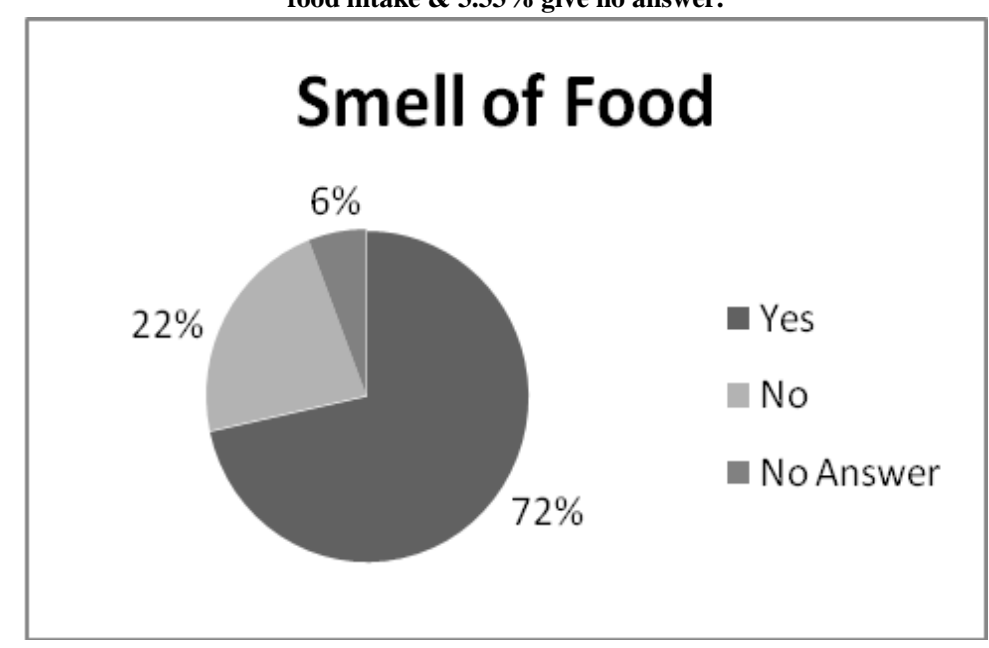

Figure 2: The figure shows $72 \%$ people influence by smell of food, $22.60 \%$ individuals were not influenced by the smell of food while $6 \%$ give no answer 


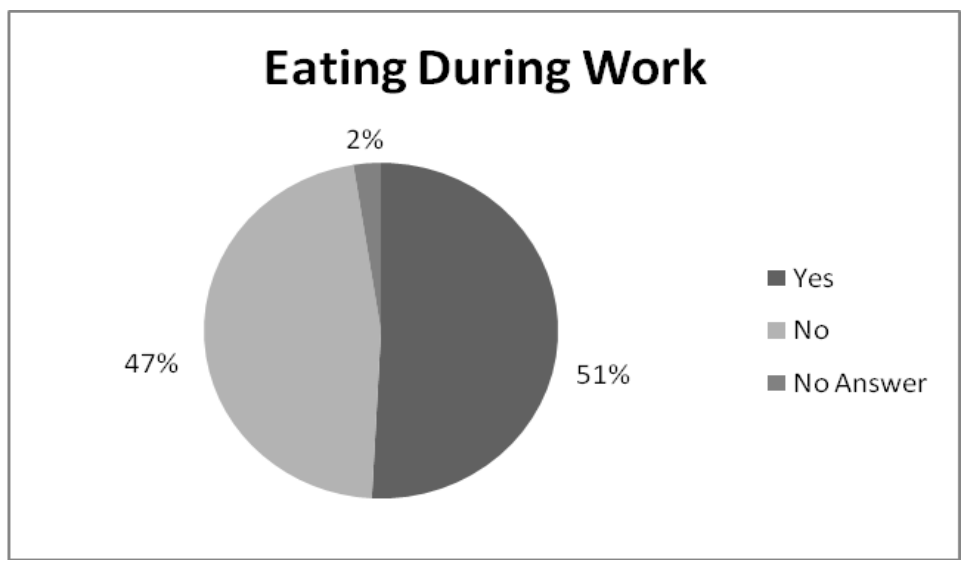

Figure 3: The figure shows the high prevalence rate of eating during work hours i.e. $51 \%$ whereas $47 \%$ avoid eating during working while $2 \%$ give no answer.

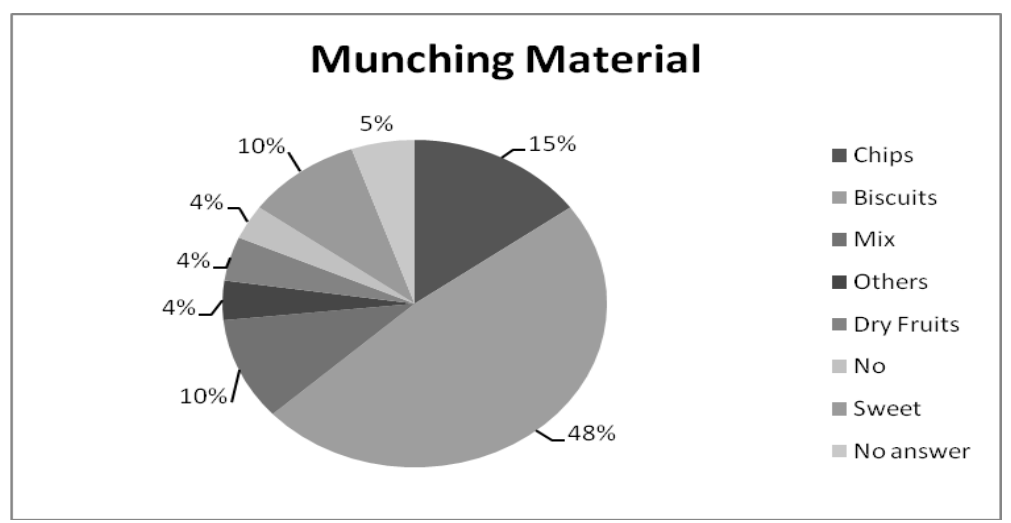

Figure 4: This figure represents munching habit of 120 individuals, $48 \%$ eat biscuits while $15.83 \%$ consume chips as their munching material, $4 \%$ do not eat, $10 \%$ rely on sweets , $4 \%$ use dry fruits , $10 \%$ use Mix items , $4 \%$ has other preferences and rest $4 \%$ give no answer.

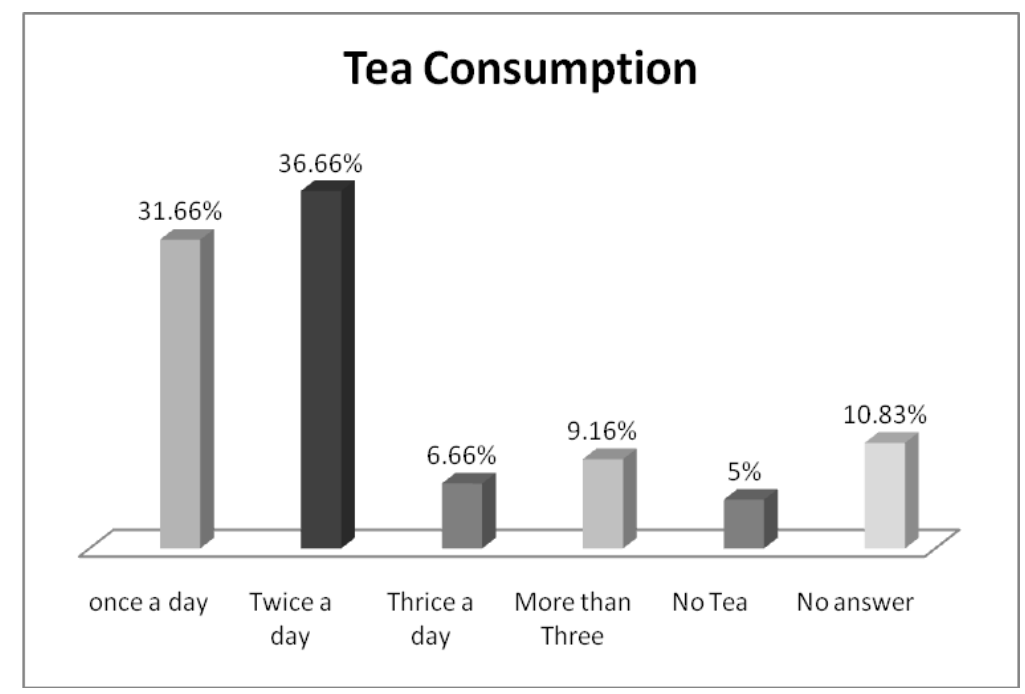

Figure 5: The graph demonstrates routine tea intake of which $31 \%$ have tea once a day, $37 \%$ have twice a day, $7 \%$ have thrice a day, $9 \%$ have tea more than three time a day whereas $5 \%$ take no tea and $11 \%$ give no answer. 


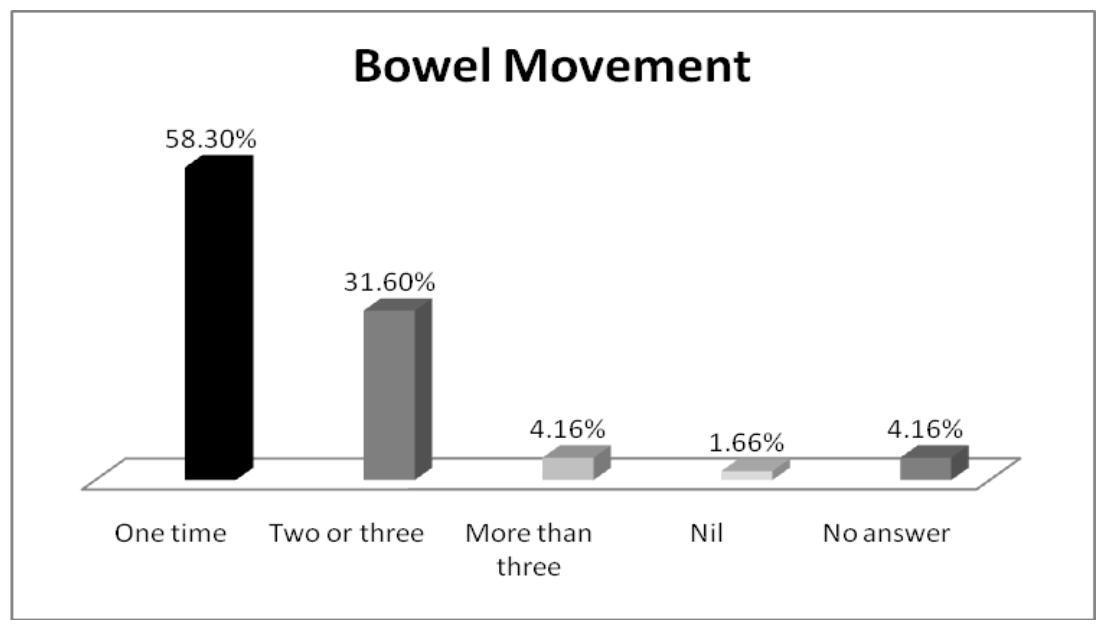

Figure 6: The above graph is a representation of bowel movement variations, out of 120 individuals $58.30 \%$ experience once a day, $31.60 \%$ go through twice or thrice a time, $4.16 \%$ has more than three times, while $4.16 \%$ give no answer.

\section{DISCUSSION}

The limited knowledge of nutrition and healthy food are the factor that leads to cause eating disorder(Anne, 2009) while binge eating is common among overweight and obese people mostly looking for weight loss treatment and there is some indication that it is associated with poor treatment fulfillment(Any, 2009).Eating disorders are multifaceted conditions caused by a combination of biological, socio-cultural, psychological, family and precipitating factors disappointment with body image and unhealthy eating behaviors are important issues for mainly in female, exposure of media has also been concerned with enhancing risk for the development of an eating disorder no one want poor body image and cultural pressure to be a thin (Zain, 2011). Strike dieting or food restriction have been verified to surge the risk of binge eating and prolong binge eating causes binge eating disorder. Physiologically craving is all about the wish for food triggered by anything from the thought, smell and sights of it a powerful desire for one particular type of food. Taste, satisfaction or pleasure of food is determined by the opioid system Ingestion of sweet and fatty foods and fluids increases opioid receptor binding within this region of the natural reward system and by the system gammaaminobutric acid/benzodiazepine neurotransmitters (Mathes, 2009). On the other hand, the desire for food is determined by the mesencephalic dopaminergic system. People can eat being full so there may be other motives to eat beyond hunger. This suggests a second system involved in food motivation, namely motivational or reward system (Nilofer, 2006 ; Ignacio, 2012). However the function of sweet in regulation of appetite is not fully cleared though some studies show a stimulating effect and most studies show no effect of sweet taste on appetite and food intake (Sorensen, 2003). Whereas eating disorders can also cause several system diseases like liver dysfunction, osteoporosis, and diabetic complications to acrocyanosis. Particularly, anorectic patients have been reported to die at a premature age possibly from one of the above stated medical complications (Mitchell, 2006) the previous research had also shown that eating disorders run in families. Behavioral symptoms or incidence of binge eating is found in our population which could lead to cause eating disorder. For the prevention the important aspect of controlling binge eating is to find alternate ways to handle stress, eat three times meals a day plus healthy snacks avoid the temptation by clearing your fridge and cupboards of your favorite binge foods. Stop dieting, exercise will help you lose weight in a healthy way Learn to distinguish between physical and emotional hunger. Write down what you eat, when, how much, and how you're feeling when you eat. You may see patterns emerge that reveal the connection between your moods and binge eating. The limitation of our study that findings from this study are all based on self-report and we have focused mostly female's participants. Further study should be conducted by the help of Diagnostic and Statistical Manual of Mental Disorders (DSM) criteria, clinical interview, the Eating Attitudes Test, Eating Disorders Inventory, Body Shape Questionnaire, and others can be used to assess eating disorders.

\section{CONCLUSION}

The results support the incidence of eating disorder in our population is present because of binge eating. However, serious consideration in the identification, treatment and management of the eating disorders binge eating, bulimia nervosa and related conditions, planning high quality care for those with eating disorders emphasizing the importance of the experience of care for patients.

\section{REFERENCE}

- Abraham, S. (2008). Eating disorders: The facts. Oxford University Press.

- Amy, A. G., Heather, M. N., Patricia, H., Mace, C., Cralen, D., Vicki, G. D., Marci, E. G., Thomas, A. W., Delia, S. W., Donald, W., \& Susan, Z. Y. (2008). Binge Eating and Weight Loss Outcomes in Overweight and Obese Individuals With Type 2 Diabetes. Arch Gen Psychiatry, 65 (12).

- Anju, P. (2012) Eating Disorders Among School Going Children Of Himachal Pradesh. International Journal Of Behavioral Social And Movement Sciences, 1(3), 94-99.

- $\quad$ Anne, K., Sonja, S., \& Joachim, W. (2009). Eating behaviour and eating disorders in students of nutrition sciences. Public Health Nutrition, 13(1), 32-37. 
- Anthony, P. W., Elizabeth, H., \& Neema, J. (2005) Neuropsychiatric effects of caffeine. Advances in Psychiatric Treatment, 11(6), 432-439

- $\quad$ Cepeda-Benito, A., Fernandez, M. C., \& Moreno, S. (2003). Relationship of gender and eating disorder symptoms to reported cravings for food: construct validation of state and trait craving questionnaires in Spanish. Appetite, 40(1), 4754.

- $\quad$ Byron, R.C., Clark, M. M., Pera, V., Niaura, R. S., \& Abrams, D. B. (1999). Binge eating, body image, depression, and self-efficacy in an obese clinical population. Obesity Research, 7(4), 379-386.

- Christopher, G. F., Doll, H. A., Welch, S. L., Hay, P. J., Davies, B. A., \& O'Connor, M. E. (1998). Risk factors for binge eating disorder: a community-based, case-control study. Archives of general psychiatry, 55(5), 425-432.

- Lowe, M. R. (1993). The effects of dieting on eating behavior: a three-factor model. Psychological bulletin, 114(1), 100

- Davies, G. J., Crowder, M., Reid, B., \& Dickerson, J. W. (1986). Bowel function measurements of individuals with different eating patterns. Gut, 27(2), 164-169.

- $\quad$ Doris, A., Klump, K. L., Thornton, L., Brandt, H., Crawford, S., Fichter, M. M. \& Bulik, C. M. (2006). Smoking in eating disorders. Eating Behaviors, 7(4), 291-299.

- Heather, A. W. (2001). Binge Eating As A Means For Evading Identity Issues: The Association Between An Avoidance Identity Style And Bulimic Behavior. International Journal of Theory And Research, 1(2), 161178.

- Ignacio, J.L. (2012). Neurophysiological Basis of Food Craving. State of the art of therapeutic, 29. 29-44

- $\quad$ Kuehnel, B.H., Wadden, T.A.(1994) Binge eating disorder, weight cycling, and psychopathology. Int $\mathrm{J}$ Eating Disord. 15(4): 321-329.
- $\quad$ Mathes, W. F., Brownley, K. A., Mo, X., \& Bulik, C. M. (2009). The biology of binge eating. Appetite, 52(3), 545553.

- $\quad$ Mitchell, J., \& Crow, S. (2006). Medical complications of anorexia nervosa and bulimia nervosa. Curr Opin Psychiatry, 19(4), 438-443

- $\quad$ Nilofer, F. S. (2006) Thinness: a woman's conflict or Eating Disorders: Facts and the Search for Solutions, J Pak Med Assoc, 56(8), 345-346.

- $\quad$ Peter, J.C., \& Fairburn, C. G. (1983). Binge-eating and selfinduced vomiting in the community. A preliminary study. The British Journal of Psychiatry, 142(2), 139-144.

- $\quad$ Shirley, A. A. B., Curry, S. J., Kristal, A. R., Lazovich, D., Feng, Z., \& Wagner, E. H. (1997). A dietary intervention in primary care practice: the Eating Patterns Study. American journal of public health, 87(4), 610-616.

- $\quad$ Sorensen, L.B., Moller, P., Flint, A., Martens, M., \& Raben, A. (2003). Effect of sensory perception of foods on appetite and food intake: a review of studies on humans, International Journal of Obesity, 27, 1152-1166

- Stunkard, A. J. (1959) Eating patterns and obesity. Psychiatric Quarterly, 33, 284-295.

- $\quad$ Mathes, W. F., Brownley, K. A., Mo, X., \& Bulik, C. M. (2009). The biology of binge eating. Appetite, 52(3), 545553.

- Zain, A., Abdul, L., Shanza, K., \& Waqas, F. (2011). Impact of Media on Development of Eating Disorders in Young Females of Pakistan. International Journal of Psychological Studies, 3(1), 122-147.

- $\quad$ Zali, Y., \& Jennifer, O. (2008). Prevention program of body image \& eating disorder on university campuses: a review of large controlled intervention. Health promotion internal, 23(2), 173-189. 